OPEN ACCESS

Edited by: Pasquale Pisapia,

University of Naples Federico II, Italy

Reviewed by:

Alessandro Russo,

A. O. Papardo, Italy

Ilaria Attili,

European Institute of Oncology

(IEO), Italy

*Correspondence:

Xiang Yan

yxiang301@sina.com

Yi Cai

yicai108@gmail.com

Specialty section:

This article was submitted to Pharmacology of Anti-Cancer Drugs,

a section of the journal

Frontiers in Oncology

Received: 27 August 2021 Accepted: 07 October 2021

Published: 25 October 2021

Citation:

Zhi X, Luo J, Li W, Wang J, Wang Y, Cai $Y$ and Yan $X$ (2021) Case Report: Osimertinib Followed by Osimertinib Plus Bevacizumab, Personalized Treatment Strategy for a Lung Cancer Patient

With a Novel EGFR Exon 20 Insertion D770_N771insGT and Multiple Brain Metastases.

Front. Oncol. 11:733276. doi: 10.3389/fonc.2021.733276

\section{Case Report: Osimertinib Followed by Osimertinib Plus Bevacizumab, Personalized Treatment Strategy for a Lung Cancer Patient With a Novel EGFR Exon 20 Insertion D770_N771insGT and Multiple Brain Metastases}

\author{
Xiaoyu Zhi ${ }^{1,2}$, Jiancheng $\mathrm{Luo}^{3}$, Weiwei $\mathrm{Li}^{4}$, Jinliang Wang ${ }^{1}$, Yuying Wang ${ }^{1,2}$, \\ $\mathrm{Yi} \mathrm{Cai}^{5 *}$ and Xiang Yan ${ }^{1 *}$ \\ ${ }^{1}$ Department of Oncology, Chinese People's Liberation Army (PLA) General Hospital, Beijing, China, ${ }^{2}$ Medical School of \\ Chinese People's Liberation Army (PLA), Beijing, China, ${ }^{3}$ Aiyi Technology Co., Ltd, Beijing, China, ${ }^{4}$ Department of Oncology, \\ The 81st Group Army Hospital of Chinese People's Liberation Army (PLA), Zhangjiakou, China, ${ }^{5}$ Independent Researcher, \\ Ellicott City, MD, United States
}

Epidermal growth factor receptor (EGFR) tyrosine kinase inhibitors (EGFR-TKIs) are the standard of care for non-small cell lung cancer (NSCLC) patients with EGFR exon 19 deletion and L858R mutations. However, no EGFR TKI has been approved for NSCLC patients harboring insertion mutations in EGFR exon 20 (EGFRex20ins), a subgroup of uncommon EGFR mutations resistant to first-generation EGFR TKIs. This unmet clinical challenge is further complicated by disease progression due to brain metastases (BMs), which limits the use of EGFR TKIs with low intracranial activity. Osimertinib, a thirdgeneration EGFR TKI with high CNS activity, has demonstrated superior efficacy as a firstline treatment for EGFR-mutant NSCLC with or without BM. The VEGF pathway is a key mediator of cancer metastasis and resistance to EGFR TKIs. Accumulating evidence has demonstrated that the addition of anti-VEGF agents to EGFR TKIs provides an alternative treatment option for the clinical management of EGFR-mutant NSCLC. We herein report an NSCLC case with a novel EGFRex20ins mutation D770_N771insGT and multiple brain metastases who briefly responded to first-line osimertinib treatment and subsequently achieved prolonged disease control with osimertinib plus bevacizumab as second-line treatment. Our case suggests that osimertinib in combination with bevacizumab may be an effective option for NSCLC patients with specific EGFRex20ins mutations and brain metastases.

Keywords: osimertinib, bevacizumab, exon 20 insertion mutation, non-small cell lung cancer, brain metastasis 


\section{INTRODUCTION}

Epidermal growth factor receptor (EGFR) mutations are major drivers of non-small cell lung cancer (NSCLC) (1). Common EGFR mutations (exon 19 deletion and L858R) and some uncommon mutations (G719X, S768I, L861Q) are sensitive to EFGR tyrosine kinase inhibitors (TKIs) (2). In contrast, EGFR exon 20 insertion (EGFRex20ins) mutations represent a heterogeneous group of uncommon EGFR mutations generally associated with a lack of response to EFGR TKIs (2).

The molecular heterogeneity of EGFRex20ins mutations is largely mediated by three elements: position, length, and the exact amino acid sequence of the insertion $(3,4)$. Approximately $87 \%$ of EGFRex20ins mutations are clustered in a span of 14 amino acids containing two motifs: the $\alpha$ C-helix (E762_M766) and the $\alpha C-\beta 4$ loop (A767_C775) (4). About 5\% of EGFRex20ins insertions are located at the $\mathrm{N}$-terminus of the $\alpha \mathrm{C}$-helix (A763_Y764insX), which is sensitive to EGFR TKIs. In contrast, EGFRex20ins mutants with insertions in the $\alpha \mathrm{C}-\beta 4$ loop have an unaltered ATP-binding pocket and are intrinsically resistant to first-generation EGFR TKIs. In 2017, Kohsaka et al. developed a mixed-all-nominated-mutants-in-one (MANO) method to evaluate the drug sensitivity of 101 EGFR mutants to five EGFR TKIs (gefitinib, erlotinib, afatinib, osimertinib, and rociletinib). This work showed that five EGFRex20ins mutants included in this study (S768_D770dup/D770_N771insSVD, N771_P772insN, H773_V774insH, H773_V774insPH, V774_C775insHV) are resistant to gefitinib, erlotinib, and rociletinib but display some varied sensitivity to afatinib and osimertinib (5). Furthermore, there was significant variation in the drug sensitivity of these five mutants as N771_P772insN was much more sensitive to afatinib/osimertinib than the others.

When EGFR TKIs are considered a treatment option for EGFRex20ins-mutant NSCLC, physicians need to find the right EGFR TKI with high activity for EGFR mutants and selectivity over wild-type (WT) EGFR. A recent study showed that mobocertinib (TAK-788) inhibits the 4 most common EGFRex20ins mutants (A767_V769dup, S768_D770dup, D770_N771insNPG, and N771_ H773dup) more potently than WT EGFR while afatinib showed the opposite pattern (6). Interestingly, osimertinib has selectivity for D770_N771insNPG but not the others. Given the heterogeneity of EGFRex20ins mutations, the practice of personalized therapy in patients with these mutations requires a detailed analysis of specific variants.

The VEGF pathway is a key regulator of cancer metastasis and anti-VEGF therapies have been approved for use in NSCLC (7). Preclinical studies have found that VEGF and EGFR pathways share common downstream signaling, and their crosstalk can promote disease progression $(8,9)$. Recent clinical trials have demonstrated that the addition of anti-VEGF therapy to erlotinib in treatment-naive patients with EGFR-mutant NSCLC significantly improved their clinical outcomes $(10,11)$. Based on these results, the NCCN guidelines include erlotinib plus bevacizumab or ramucirumab as first-line treatment options for NSCLC patients with common EGFR mutations (2). However, whether EGFR/VEGF dual blockade could improve the clinical outcome of EGFRex20ins-mutant NSCLC patients is unknown.
Here, we present an NSCLC patient with a novel EGFRex20ins mutation and brain metastases who achieved durable disease control with osimertinib plus bevacizumab after a brief response to first-line osimertinib monotherapy.

\section{CASE DESCRIPTION}

In January 2019 a 69-year-old Chinese male ex-smoker who had had type 2 diabetes for 10 years presented with dizziness and unsteady walking for two months. Computed tomography (CT) scans of the chest revealed two masses and multiple nodules in the right lower lobe. Brain magnetic resonance (MR) imaging found multiple metastases and ECT showed enhanced radioactivity at $\mathrm{T} 2$ and $\mathrm{L} 4$. The patient was diagnosed with stage IV (T3N0M1) lung adenocarcinoma via percutaneous biopsy. Next-generation sequencing (NGS) testing showed an EGFR exon20 insertion (p.D770-N771insGT) mutation with concurring ERBB2 and TP53 mutations (Figure 1). According to the American College of Medical Genetics and Genomics (ACMG) guidelines (12), the ERBB2 P1170A and TP53 R197_V197insA mutations were classified as benign and a variant of unknown significance (VUS), respectively. Therefore, they were excluded from the treatment decision-making process.

The patient refused chemotherapy and whole-brain radiation, the standard care for his condition. As D770_N771insGT is a novel EGFRex20ins mutant, we used data from two very similar mutants as the reference to guide our EGFR TKI selection process. For the D770_N771insNPG mutant, the IC50 values of erlotinib, afatinib, and osimertinib were $1146 \mathrm{nM}, 43 \mathrm{nM}$, and $42 \mathrm{nM}$, respectively (13). The selectivity indexes (log10 [IC50mutant/IC50Wild Type]) for afatinib and osimertinib were above zero and below -1 , which means osimertinib has a better therapeutic dose window than afatinib for this variant. Importantly, Piotrowska et al. reported an 11- month-long clinical response to osimertinib in a metastatic lung cancer patient harboring an EGFR S768_D770dup mutation (14). Additionally, preclinical comparison of osimertinib with other EGFR TKIs in an EGFR-mutant NSCLC brain metastasis model showed that osimertinib has greater penetration of the bloodbrain barrier than gefitinib, rociletinib, or afatinib at clinically relevant doses (15). The brain:plasma Cmax ratios for osimertinib, afatinib, gefitinib, and rociletinib were $3.41,<0.36$, 0.21 , and $<0.08$, respectively. Based on all these data, the patient was administered osimertinib (80 mg daily). It was well tolerated with only grade 1 nausea and his symptoms disappeared 20 days later. At the 4 month follow-up, the lesions on the right lung and brain achieved partial response by RECIST (version $1.1 ;-37.5 \%$ and $-52.3 \%$ response, respectively). At the end of May, the patient complained about headache, and progression of the brain lesion was noted by MRI. With the consent of the patient, bevacizumab (400 $\mathrm{mg} / \mathrm{month}$ ) was added to osimertinib from June $6^{\text {th }}, 2019$. The combination was well tolerated without any report of toxicity. CNS symptoms cleared up again as the brain nodule stopped growing and edema was eliminated. Strikingly, the lung lesion achieved PR again (version $1.1 ;-82.2 \%$ response) with the combination 


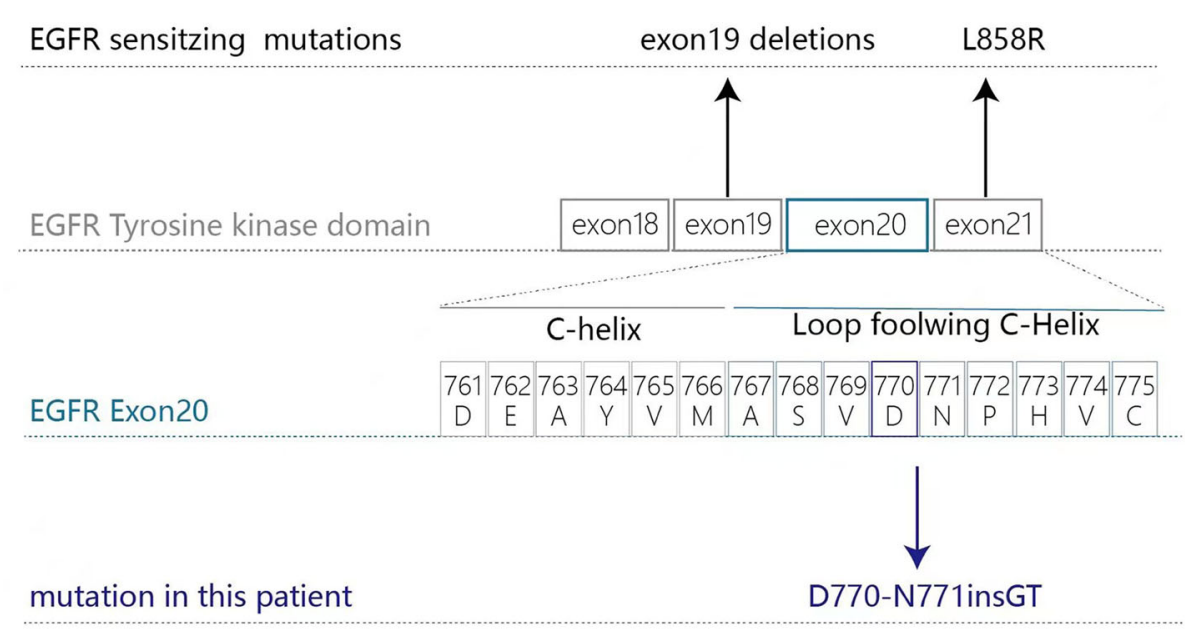

FIGURE 1 | Gene check analysis showed that the patient had an EGFR exon20 insertion (p.D770-N771insGT) mutation.

therapy. At follow-up 2.1, 4.5, 6.5, and 9.1 months after the combination therapy, the patient had an ongoing clinical benefit and stable disease. The dynamic changes in chest CT and brain MRI during treatments are shown in Figure 2.

Two months before the patient's death, there was a significant decline in his physical activity and consciousness. On March 5th, a CT scan of the chest revealed no progressive disease. Brain MRI was not feasible due to his poor physical condition. Laboratory tests showed hemoglobin $96 \mathrm{~g} / \mathrm{L}$ (normal range, $137-179 \mathrm{~g} / \mathrm{L}$ ), creatinine $177.7 \mathrm{umol} / \mathrm{L}$ (normal range, 30-110 umol/L), brain natriuretic peptide precursor (proBNP) $234.9 \mathrm{pg} / \mathrm{mL}$ (normal range, $0-150 \mathrm{pg} / \mathrm{mL}$ ), carcinoembryonic antigen (CEA) $22.46 \mathrm{ug} /$ $\mathrm{L}$ (normal range, 0-5.0 ug/L), CYFRA21-1 $5.15 \mathrm{ng} / \mathrm{mL}$ (normal range, 0.1-4.0 ng/mL), and SCC $3.1 \mathrm{ng} / \mathrm{mL}$. Bevacizumab was discontinued due to the increased creatine level, a biomarker for kidney damage. Elevated proBNP levels are a heart failure

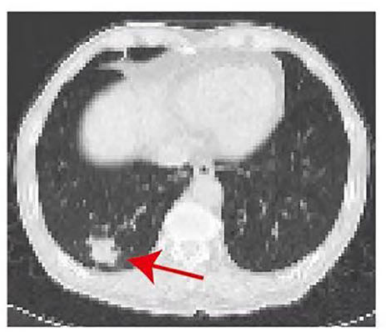

2019-01-04

Baseline CT

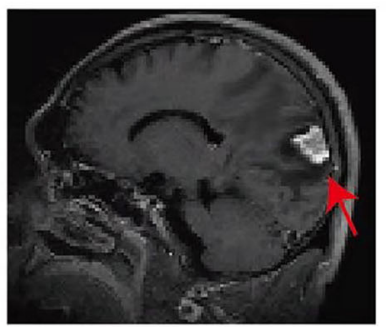

2019-01-03

Baseline MRI

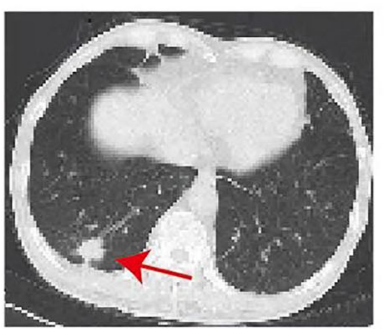

2019-03-23

Stable disease

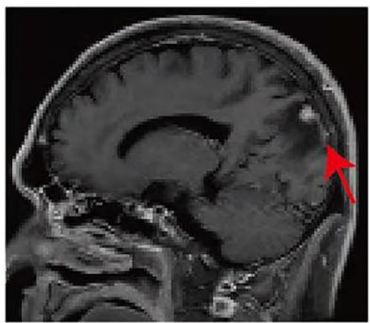

2019-03-24

Partial response

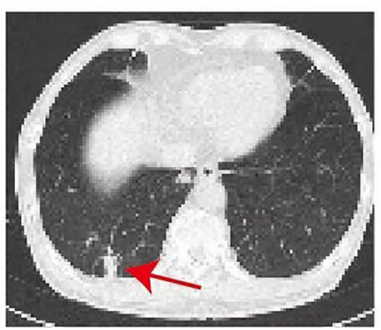

2019-06-27

Partial response

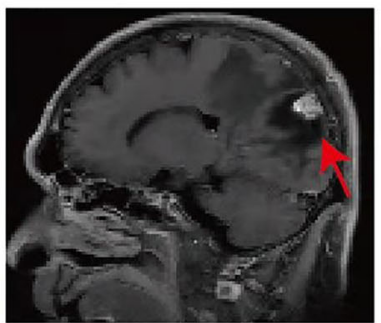

2019-06-01

Progressive disease

FIGURE 2 | Patient dynamic changes in the chest CT and brain MRI during treatments. 
biomarker related to cardiac adverse events associated with osimertinib. Because of his physical condition and the lack of symptoms of heart failure, the patient continued with osimertinib. He was discharged after his symptoms improved. Although we suspected the presence of leptomeningeal metastasis, we could not confirm this diagnosis with enhanced brain MRI or physical examination. Due to poor physical condition, the patient declined lumbar puncture for further diagnosis. Therefore, the exact cause of his death is unknown. On March 20th, the patient died with an overall survival time of 13.5 months. Figure 3 shows a flow pathway for treatment and assessment.

\section{DISCUSSION}

Activating EGFR mutations are common drivers of NSCLC (1). EGFR exon 19 deletions and the L858R mutation represent 85$90 \%$ of kinase domain mutations observed in NSCLC and are sensitive to EGFR tyrosine kinase inhibitors (TKIs) (16). In contrast, uncommon EGFRex20ins mutations are generally resistant to early-generation EGFR TKIs (17). Currently, no EGFR TKI has been approved for EGFRex20ins-driven NSCLC and the standard of care for this condition is chemotherapy associated with 50-63\% response rates and median PFS 4.1-6.4 months (18-20). Therefore, there is a substantial clinical need to improve the outcome of this NSCLC patient subgroup.

Currently, osimertinib is the preferred first-line EGFR TKI for NSCLC patients with sensitizing EGFR mutations (2). However, its efficacy in EGFRex20ins NSCLC has not been adequately characterized. EA5162, a single-arm, phase 2 study showed that osimertinib at $160 \mathrm{mg}$ achieved a response rate of $25 \%$, disease control rate of $85 \%$, and mPFS of 9.7 months in 20 EGFRex 20 ins
NSCLC patients (21). In a single-center retrospective study of osimertinib for 62 EGFRex20ins NSCLC patients, osimertinib 80 $\mathrm{mg}$ or $160 \mathrm{mg}$ resulted in a response rate of $6.5 \%$, disease control rate of $53.2 \%$, and mPFS of 2.3 months (22). There was no significant difference in median PFS between patients who received osimertinib $80 \mathrm{mg}$ or $160 \mathrm{mg}$ (2.5 vs. 1.3 months, $\mathrm{P}=0.161)$.

Because EGFRex20ins mutants are heterogeneous in their drug sensitivity, the selection of EGFR TKIs targeting specific EGFRex20ins mutants requires a good understanding of their mechanism of activation and effects on drug binding. For instance, steric hindrance in the drug-binding pocket of some EGFRex20ins mutants restricts the binding of EGFR TKIs with a rigid core and large terminal group such as osimertinib (6). Under this condition, afatinib can have a better inhibition potential than osimertinib. Consistently, the average IC50 values for afatinib and osimertinib across 6 EGFRex20ins mutants (A767insASV, S768dupSVD, V769insASV, D770insNPG, D770insSVD, H773insNPH) were $39.9 \mathrm{nM}$ and $103 \mathrm{nM}$, respectively (23). And afatinib achieved a durable response in a small number of NSCLC patients harboring specific EGFRex20ins mutations (24-27). Similarly, poziotinib has a less rigid core and smaller terminal groups than osimertinib, which allows it to assess the restricted drugbinding pocket of EGFRex20ins mutants more easily (16). In the ZENITH20-3 phase 2 trial, EGFRex20ins NSCLC patients receiving poziotinib had an ORR of $27.8 \%$ (95\% confidence interval [CI] 18.4-39.1\%) and a median PFS of 7.2 months (28). Mobocertinib (TAK-788) is a novel EGFR TKI rationally designed to fit the drug-binding pocket of EGFRex20ins mutants which results in increased affinity compared with osimertinib (6). In a phase $1 / 2$ trial, pretreated EGFRex20ins NSCLC patients receiving mobocertinib achieved an ORR of $28 \%$ and a median

January 2019

Mutation detected

lung adenocarcinoma diagnosed

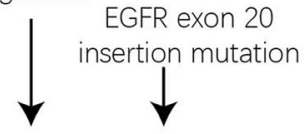

20 March 2020

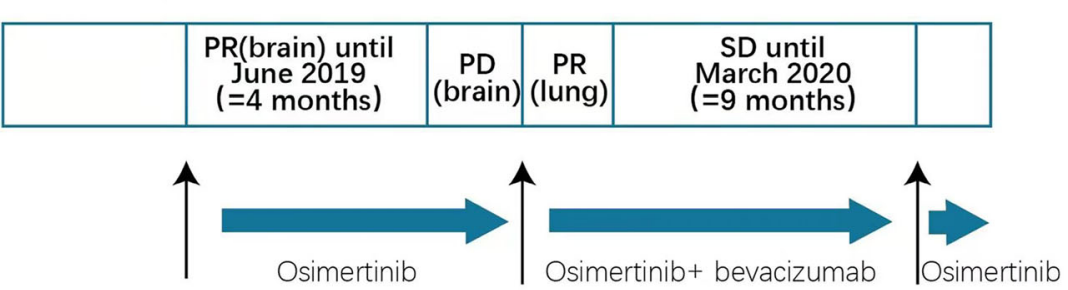

30 January 2019

6 June 2019

5 March 2020

Treatment intervention

FIGURE 3 | Patient clinical course including treatment process and relevant assessment. 
PFS of 7.3 months (29). Interestingly, amivantamab, an EGFRMET bispecific antibody, was recently approved to treat EGFRex20ins NSCLC patients (30). This approval was based on the results of a phase 1 trial CHRYSALIS (NCT02609776) in which amivantamab achieved an ORR of $40 \%$ (95\% CI: $29 \%$, $51 \%)$ and a median response duration of 11.1 months (95\% CI: 6.9, not evaluable) (31).

Preclinical studies have found that the crosstalk between the VEGF and EGFR pathways plays an important role in the pathogenesis and metastasis of EGFR-mutant NSCLC $(8,9)$. Activation of EGFR signaling increases VEGF expression through hypoxia-independent mechanisms, and elevated VEGF, in turn, contributes to resistance to EGFR TKIs (9). Therefore, dual EGFR-VEGF inhibition may provide greater antitumor activity than the respective monotherapies (7). Indeed, the NEJ026 and ARTEMIS-CTONG1509 trials showed that PFS was significantly improved in the erlotinib plus bevacizumab group compared with the erlotinib group (16.9 versus 13.3 mo, HR: $0.605, \mathrm{p}=0.016 ; 17.9$ vs. 11.2 mo, HR: $0.55, \mathrm{p}$ $<0.001$, respectively) $(10,32)$. The RELAY trial evaluated the efficacy of erlotinib plus ramucirumab versus erlotinib plus placebo (11). PFS was superior in the combination group compared with the control group (median PFS: 19.4 versus 12.4 mo, HR: 0.59, p < 0.0001). Currently, the NSCLC NCCN guidelines include erlotinib plus bevacizumab or ramucirumab as first-line treatments for patients with sensitizing EGFR mutations (2).

NSCLC patients with acquired erlotinib-resistant EGFR T790M mutations were excluded from most of the clinical trials mentioned above. Based on the superior efficacy of osimertinib against the T790M mutation and positive results of the RELAY and NEJ026 trials, it is expected that osimertinib plus bevacizumab may achieve better clinical efficacy than osimertinib alone in T790M-positive NSCLC patients. However, data from the randomized WJOG 8715L phase 2 trial revealed that this combination did not improve PFS or OS in T790M-positive NSCLC (33).

Here, we conducted a three-step analysis to reconcile the conflicting results between the WJOG 8715L trial and previous erlotinib/bevacizumab combination trials. First, we separated OS results from PFS results. Since no OS benefit was seen in phase 3 trials for erlotinib plus bevacizumab in EGFR-mutant NSCLC (7), one should not expect osimertinib plus bevacizumab to achieve longer OS than osimertinib alone. Second, we separated the first-line setting from the second-line setting in the PFS analysis. In the WJOG 8715L trial, the median PFS of second-line bevacizumab plus osimertinib was numerically shorter than that of osimertinib alone (9.4 vs. 13.5 months, $\mathrm{p}=$ 0.2 ). However, in the first-line setting, this combination achieved a median PFS of 19 months in a phase $1 / 2$ trial conducted at MSKCC, close to the median PFS of 18.9 months with that of osimertinib alone in the FLAURA trial $(34,35)$. It is noteworthy that more patients in the MSKCC trial had brain metastases than those in the FLAURA trial (31\% vs. 19\%), which may influence PFS. Third, we identified multiple putative confounding factors in the WJOG 8715L trial. The percentages of stage IV diseases, prior chemotherapy, prior anti-VEGF therapy, and brain metastases in the combination group were all higher than those in the osimertinib group ( $83 \%$ vs. $63 \%, 25 \%$ vs. $17 \%$, $20 \%$ vs. $10 \%, 30 \%$ vs. $22 \%$ ) (33). Although these confounding factors were not statistically significant individually, all four factors were in favor of the osimertinib group. Furthermore, subgroup analysis revealed that patients with prior anti-VEGF therapy had a significantly shorter PFS than those who did not in the combination group (4.6 vs. 11.1 months; HR, $0.41 ; P=.03$ ) but not in the osimertinib group (15.1 months vs. 13.7 months; $\mathrm{HR}, 1.19 ; P=.85$ ) (33). Given the relatively small sample size (n $=81$ ) and imbalanced patient characteristics of the WJOG $8715 \mathrm{~L}$ trial, it will be too early to draw conclusions regarding the efficacy of osimertinib plus bevacizumab before the results of more trials become available.

In line with our analysis, the results of the ETOP BOOSTER phase 2 trial with a setting identical to the WJOG 8715L trial and larger sample size $(n=155)$ revealed that osimertinib plus bevacizumab yielded significantly longer median PFS in current/former smokers than osimertinib alone (16.5 vs. 8.4 months, $\mathrm{p}=0.0052$ ) (36). More importantly, the combination also achieved significantly longer median OS than osimertinib alone in the smoker subgroup. These results indicate that osimertinib plus bevacizumab could benefit the smoker subgroup of EGFR-mutant NSCLC patients.

One unresolved question is whether EGFRex20ins-driven NSCLC will share similar downstream signaling profiles and vulnerabilities that can be exploited therapeutically with dual inhibition of EGFR and VEGF signaling. Interestingly, a preclinical study showed that three representative EGFRactivating mutations (exon 19 deletion, L858R, N771delinsFH) induce HIF-1 $\alpha$ expression, which in turn upregulates VEGF expression (9). Osimertinib treatment reduced HIF- $1 \alpha$ and VEGF in L858R or exon 19 deletion mutant cells while poziotinib treatment decreased HIF- $1 \alpha$ and VEGF levels in N771delinsFH mutant cells. These results indicated that abnormal EGFR signaling activation was the dominant driver of VEGF upregulation in NSCLC cells and that EGFR-dependent upregulation of VEGF can be targeted with EGFR TKIs. Furthermore, in two PDXs harboring EGFRex20ins mutations (H773insNPH and S768dupSVD), poziotinib plus bevacizumab resulted in near-complete tumor regression and significantly prolonged PFS compared with either reagent alone (9). These results indicated that the addition of bevacizumab to EGFR TKIs may achieve better therapeutic efficacy in EGFRex20ins NSCLC than EGFR TKIs alone.

Accumulating evidence suggests that EGFR TKIs plus bevacizumab are a promising treatment option for EGFRmutant NSCLC patients with CNS diseases. Data from the ARTEMIS-CTONG1509 phase 3 trial showed that the addition of bevacizumab to erlotinib significantly improved PFS in patients with brain metastases (32). Compared to erlotinib alone, the combination also had a positive trend to prolong OS in this patient subgroup. Similarly, in the MSKCC phase 2 trial, osimertinib plus bevacizumab resulted in a $100 \%$ response rate in 6 patients with brain metastases, including two complete 
responses (35). Interestingly, this combination also demonstrated CNS activity in patients with leptomeningeal metastases $(37,38)$. Consistently, the addition of bevacizumab to osimertinib achieved significantly longer PFS than osimertinib alone in our patient, who had multiple brain metastases.

\section{CONCLUSIONS}

Our case suggests that osimertinib alone or in combination with bevacizumab can be a feasible therapeutic option for NSCLC patients with specific EGFRex20ins mutations and brain metastases. Future studies are required to determine the efficacy and safety of this combination in NSCLC patients with different EGFRex20ins mutations.

\section{DATA AVAILABILITY STATEMENT}

The original contributions presented in the study are included in the article/supplementary files. Further inquiries can be directed to the corresponding authors.

\section{ETHICS STATEMENT}

Ethical review and approval was not required for the study on human participants in accordance with the local legislation and institutional requirements. The patients/participants provided their written informed consent to participate in this study. Written informed consent was obtained from the individual(s)

\section{REFERENCES}

1. Eric A, Mara R, Miruna B, Angela H, Katherine AH, Leslie C, et al. Comprehensive Molecular Profiling of Lung Adenocarcinoma. Nature (2014) 511(7511):543-50. doi: 10.1038/nature13385

2. Ettinger DS, Wood DE, Aisner DL, Akerley W, Bauman JR, Bharat A, et al. NCCN Guidelines Insights: Non-Small Cell Lung Cancer, Version 2.2021. J Natl Compr Canc Netw (2021) 19(3):254-66. doi: 10.6004/jnccn.2021.0013

3. Friedlaender A, Subbiah V, Russo A, Banna GL, Malapelle U, Rolfo C, et al. EGFR and HER2 Exon 20 Insertions in Solid Tumours: From Biology to Treatment. Nat Rev Clin Oncol (2021) 77(10):2712-21. doi: 10.1038/s41571021-00558-1

4. Meador CB, Sequist LV, Piotrowska Z. Targeting EGFR Exon 20 Insertions in Non-Small Cell Lung Cancer: Recent Advances and Clinical Updates. Cancer Discovery (2021) 11(9):2145-57. doi: 10.1158/ 2159-8290.Cd-21-0226

5. Kohsaka S, Nagano M, Ueno T, Suehara Y, Hayashi T, Shimada N, et al. A Method of High-Throughput Functional Evaluation of EGFR Gene Variants of Unknown Significance in Cancer. Sci Transl Med (2017) 9(416):eaan6566. doi: 10.1126/scitranslmed.aan6566

6. Gonzalvez F, Vincent S, Baker TE, Gould AE, Li S, Wardwell SD, et al. Mobocertinib (TAK-788): A Targeted Inhibitor of EGFR Exon 20 Insertion Mutants in Non-Small Cell Lung Cancer. Cancer Discov (2021) 11(7):167287. doi: 10.1158/2159-8290.Cd-20-1683

7. Le X, Nilsson M, Goldman J, Reck M, Nakagawa K, Kato T, et al. Dual EGFRVEGF Pathway Inhibition: A Promising Strategy for Patients With EGFRMutant NSCLC. J Thorac Oncol (2021) 16(2):205-15. doi: 10.1016/ j.jtho.2020.10.006 for the publication of any potentially identifiable images or data included in this article.

\section{AUTHOR CONTRIBUTIONS}

Conception and design: XY, YC. Provision of study material or patients: XZ, XY, WL, JW. Collection and assembly of data: XZ, JL. Data analysis and interpretation: XZ, JL. Manuscript writing: All authors, mainly by YC. All authors contributed to the article and approved the submitted version.

\section{FUNDING}

This study was supported by the National Natural Science Foundation of China (grant number 81902910).

\section{ACKNOWLEDGMENTS}

We are indebted to our patient who has allowed us to communicate his case.

\section{SUPPLEMENTARY MATERIAL}

The Supplementary Material for this article can be found online at: https://www.frontiersin.org/articles/10.3389/fonc.2021.733276/ full\#supplementary-material

8. Naumov GN, Nilsson MB, Cascone T, Briggs A, Straume O, Akslen LA, et al. Combined Vascular Endothelial Growth Factor Receptor and Epidermal Growth Factor Receptor (EGFR) Blockade Inhibits Tumor Growth in Xenograft Models of EGFR Inhibitor Resistance. Clin Cancer Res (2009) 15 (10):3484-94. doi: 10.1158/1078-0432.Ccr-08-2904

9. Nilsson MB, Robichaux J, Herynk MH, Cascone T, Le X, Elamin Y, et al. Altered Regulation of HIF- $1 \alpha$ in Naive- and Drug-Resistant EGFR-Mutant NSCLC: Implications for a Vascular Endothelial Growth Factor-Dependent Phenotype. J Thorac Oncol (2021) 16(3):439-51. doi: 10.1016/j.jtho.2020.11.022

10. Saito H, Fukuhara T, Furuya N, Watanabe K, Sugawara S, Iwasawa S, et al. Erlotinib Plus Bevacizumab Versus Erlotinib Alone in Patients With EGFRPositive Advanced non-Squamous non-Small-Cell Lung Cancer (NEJ026): Interim Analysis of an Open-Label, Randomised, Multicentre, Phase 3 Trial. Lancet Oncol (2019) 20(5):625-35. doi: 10.1016/s1470-2045(19)30035-x

11. Nakagawa K, Garon EB, Seto T, Nishio M, Ponce Aix S, Paz-Ares L, et al. Ramucirumab Plus Erlotinib in Patients With Untreated, EGFR-Mutated, Advanced non-Small-Cell Lung Cancer (RELAY): A Randomised, DoubleBlind, Placebo-Controlled, Phase 3 Trial. Lancet Oncol (2019) 20(12):165569. doi: 10.1016/s1470-2045(19)30634-5

12. Richards S, Aziz N, Bale S, Bick D, Das S, Gastier-Foster J, et al. Standards and Guidelines for the Interpretation of Sequence Variants: A Joint Consensus Recommendation of the American College of Medical Genetics and Genomics and the Association for Molecular Pathology. Genet Med (2015) 17(5):405-24. doi: $10.1038 /$ gim.2015.30

13. Hirano T, Yasuda H, Tani T, Hamamoto J, Oashi A, Ishioka K, et al. In Vitro Modeling to Determine Mutation Specificity of EGFR Tyrosine Kinase Inhibitors Against Clinically Relevant EGFR Mutants in non-Small-Cell Lung Cancer. Oncotarget (2015) 6(36):38789-803. doi: 10.18632/oncotarget.5887 
14. Piotrowska Z, Fintelmann FJ, Sequist LV, Jahagirdar B. Response to Osimertinib in an EGFR Exon 20 Insertion-Positive Lung Adenocarcinoma. J Thorac Oncol (2018) 13(10):e204-6. doi: 10.1016/j.jtho.2018.05.017

15. Ballard P, Yates JW, Yang Z, Kim DW, Yang JC, Cantarini M, et al. Preclinical Comparison of Osimertinib With Other EGFR-TKIs in EGFR-Mutant NSCLC Brain Metastases Models, and Early Evidence of Clinical Brain Metastases Activity. Clin Cancer Res (2016) 22(20):5130-40. doi: 10.1158/ 1078-0432.Ccr-16-0399

16. Vyse S, Huang PH. Targeting EGFR Exon 20 Insertion Mutations in nonSmall Cell Lung Cancer. Signal Transduct Target Ther (2019) 4:5. doi: 10.1038/s41392-019-0038-9

17. Yasuda H, Park E, Yun CH, Sng NJ, Lucena-Araujo AR, Yeo WL, et al. Structural, Biochemical, and Clinical Characterization of Epidermal Growth Factor Receptor (EGFR) Exon 20 Insertion Mutations in Lung Cancer. Sci Transl Med (2013) 5(216):216ra177. doi: 10.1126/scitranslmed.3007205

18. Yang G, Li J, Xu H, Yang Y, Yang L, Xu F, et al. EGFR Exon 20 Insertion Mutations in Chinese Advanced non-Small Cell Lung Cancer Patients: Molecular Heterogeneity and Treatment Outcome From Nationwide RealWorld Study. Lung Cancer (2020) 145:186-94. doi: 10.1016/ j.lungcan.2020.03.014

19. Xu CW, Wang WX, Wang D, Wang QM, Pu XX, Zhu YC, et al. PemetrexedBased Chemotherapy for non-Small-Cell Lung Cancer Patients With EGFR Exon 20 Insertion Mutation: A Multicenter Study. Transl Lung Cancer Res (2020) 9(5):1853-61. doi: 10.21037/tlcr-20-382

20. Wu JY, Yu CJ, Shih JY. Effectiveness of Treatments for Advanced Non-SmallCell Lung Cancer With Exon 20 Insertion Epidermal Growth Factor Receptor Mutations. Clin Lung Cancer (2019) 20(6):e620-30. doi: 10.1016/ j.cllc.2019.06.018

21. Piotrowska Z, Wang Y, Sequist LV, Ramalingam SS. ECOG-ACRIN 5162: A Phase II Study of Osimertinib $160 \mathrm{Mg}$ in NSCLC With EGFR Exon 20 Insertions. J Clin Oncol (2020) 38(15_suppl):9513-3. doi: 10.1200/ JCO.2020.38.15_suppl.9513

22. Yang GJ, Li J, Xu HY, Sun Y, Liu L, Li HS, et al. Osimertinib for Chinese Advanced non-Small Cell Lung Cancer Patients Harboring Diverse EGFR Exon 20 Insertion Mutations. Lung Cancer (2021) 152:39-48. doi: 10.1016/ j.lungcan.2020.11.027

23. Robichaux JP, Elamin YY, Tan Z, Carter BW, Zhang S, Liu S, et al. Mechanisms and Clinical Activity of an EGFR and HER2 Exon 20-Selective Kinase Inhibitor in non-Small Cell Lung Cancer. Nat Med (2018) 24(5):63846. doi: 10.1038/s41591-018-0007-9

24. Lin L, Wu X, Yan S, Zhu Y, Yan Z, Lv D, et al. Response to Afatinib in a Patient With NSCLC Harboring Novel EGFR Exon 20 Insertion Mutations. Onco Targets Ther (2020) 13:9753-7. doi: 10.2147/ott.S268694

25. Zöchbauer-Müller S, Kaserer B, Prosch H, Cseh A, Solca F, Bauer MJ, et al. Case Report: Afatinib Treatment in a Patient With NSCLC Harboring a Rare EGFR Exon 20 Mutation. Front Oncol (2020) 10:593852. doi: 10.3389/ fonc. 2020.593852

26. Chan RT. Afatinib for an EGFR Exon 20 Insertion Mutation: A Case Report of Progressive Stage IV Metastatic Lung Adenocarcinoma With 54 Months' Survival. Asia Pac J Clin Oncol (2018) 14(Suppl 1):7-9. doi: 10.1111/ajco.12853

27. Oyamada Y, Wada S, Fujimoto K. A Case of Advanced Lung Adenocarcinoma Harboring an Epidermal Growth Factor Receptor(EGFR) Exon 20 Insertion, D770_N771insSVD. Gan To Kagaku Ryoho (2021) 48(6):845-7.

28. Sacher A. Annals of Oncology (2021). Available at: https://www. annalsofoncology.org/article/S0923-7534(21)00060-0/fulltext (Accessed March 01, 2021)

29. Riely GJ, Neal JW, Camidge DR, Spira AI, Piotrowska Z, Costa DB, et al. Activity and Safety of Mobocertinib (TAK-788) in Previously Treated Non-
Small Cell Lung Cancer With EGFR Exon 20 Insertion Mutations From a Phase I/II Trial. Cancer Discov (2021) 11(7):1688-99. doi: 10.1158/21598290.Cd-20-1598

30. Dolgin E. Amivantamab OK'd for EGFR-Mutant NSCLC. Cancer Discov (2021) 11(7):1604. doi: 10.1158/2159-8290.Cd-nb2021-0351

31. Park K, Haura EB, Leighl NB, Mitchell P, Shu CA, Girard N, et al. Amivantamab in EGFR Exon 20 Insertion-Mutated Non-Small-Cell Lung Cancer Progressing on Platinum Chemotherapy: Initial Results From the CHRYSALIS Phase I Study. J Clin Oncol (2021) 39(30):3391-402. doi: 10.1200/jco.21.00662

32. Zhou Q, Xu CR, Cheng Y, Liu YP, Chen GY, Cui JW, et al. Bevacizumab Plus Erlotinib in Chinese Patients With Untreated, EGFR-Mutated, Advanced NSCLC (ARTEMIS-CTONG1509): A Multicenter Phase 3 Study. Cancer Cell (2021) 39(9):1279-1291.e1273. doi: 10.1016/j.ccell.2021.07.005

33. Akamatsu H, Toi Y, Hayashi H, Fujimoto D, Tachihara M, Furuya N, et al. Efficacy of Osimertinib Plus Bevacizumab vs Osimertinib in Patients With EGFR T790M-Mutated Non-Small Cell Lung Cancer Previously Treated With Epidermal Growth Factor Receptor-Tyrosine Kinase Inhibitor: West Japan Oncology Group 8715l Phase 2 Randomized Clinical Trial. JAMA Oncol (2021) 7(3):386-94. doi: 10.1001/jamaoncol.2020.6758

34. Ramalingam SS, Vansteenkiste J, Planchard D, Cho BC, Gray JE, Ohe Y, et al. Overall Survival With Osimertinib in Untreated, EGFR-Mutated Advanced NSCLC. N Engl J Med (2020) 382(1):41-50. doi: 10.1056/NEJMoa1913662

35. Yu HA, Schoenfeld AJ, Makhnin A, Kim R, Rizvi H, Tsui D, et al. Effect of Osimertinib and Bevacizumab on Progression-Free Survival for Patients With Metastatic EGFR-Mutant Lung Cancers: A Phase 1/2 Single-Group Open-Label Trial. JAMA Oncol (2020) 6(7):1048-54. doi: 10.1001/jamaoncol.2020.1260

36. Soo R. Annals of Oncology (2021). Available at: https://www.annalsofoncology.org/ article/S0923-7534(21)01148-0/fulltext (Accessed JULY 01, 2021).

37. Jiang $\mathrm{T}, \mathrm{Xu} \mathrm{X}$, Chen $\mathrm{X}$, Ding $\mathrm{N}, \mathrm{Hu} \mathrm{Q}$, Zhou C, et al. Osimertinib in Combination With Bevacizumab in EGFR-Mutated NSCLC With Leptomeningeal Metastases. Transl Lung Cancer Res (2020) 9(6):2514-7. doi: $10.21037 /$ tlcr-20-984

38. Lu ZQ, Cai J, Wang X, Wei JP, Zeng ZM, Huang L, et al. Osimertinib Combined With Bevacizumab for Leptomeningeal Metastasis From EGFRMutation non-Small Cell Lung Cancer: A Phase II Single-Arm Prospective Clinical Trial. Thorac Cancer (2021) 12(2):172-80. doi: 10.1111/17597714.13738

Conflict of Interest: Author JL was employed by company Aiyi Technology Co., Ltd.

The remaining authors declare that the research was conducted in the absence of any commercial or financial relationships that could be construed as a potential conflict of interest.

Publisher's Note: All claims expressed in this article are solely those of the authors and do not necessarily represent those of their affiliated organizations, or those of the publisher, the editors and the reviewers. Any product that may be evaluated in this article, or claim that may be made by its manufacturer, is not guaranteed or endorsed by the publisher.

Copyright (C) 2021 Zhi, Luo, Li, Wang, Wang, Cai and Yan. This is an open-access article distributed under the terms of the Creative Commons Attribution License (CC BY). The use, distribution or reproduction in other forums is permitted, provided the original author(s) and the copyright owner(s) are credited and that the original publication in this journal is cited, in accordance with accepted academic practice. No use, distribution or reproduction is permitted which does not comply with these terms. 